\title{
Teoría generalizada de la potencia instantánea aplicada a la compensación de cargas polifásicas
}

\author{
Generalized instantaneous power theory applied to multiphase loads compensation
}

\author{
Armando Jaime Ustariz-Farfan ${ }^{1} \quad$ Eduardo Antonio Cano-Plata ${ }^{1} \quad$ Hernán Emilio Tacca $^{2}$
}

Recibido 3 de septiembre de 2010, aceptado 17 de julio de 2012

Received: September 3, 2010 Accepted: July 17, 2012

\begin{abstract}
RESUMEN
El interés creciente de utilizar filtros activos de potencia para compensar las corrientes no sinusoidales producidas por las cargas no lineales ha aumentado el uso de la teoría instantánea de potencia reactiva. Sin embargo, esta teoría se puede aplicar solamente a los sistemas trifásicos de tres o cuatro conductores. Este artículo presenta una nueva definición de la potencia instantánea que puede ser aplicada a todos los sistemas polifásicos. Así, el concepto de producto tensorial es introducido para definir el tensor instantáneo de potencia y también la descomposición de la corriente en las componentes activas y reactivas sin importar el número de fases del sistema eléctrico. Finalmente, un análisis exhaustivo de casos prácticos se ha realizado a nivel de simulación, los cuales han permitido que el enfoque propuesto sea verificado.
\end{abstract}

Palabras clave: Análisis tensorial, filtros activos de potencia, potencia instantánea, producto tensorial, sistemas polifásicos.

\section{ABSTRACT}

The use of the instantaneous reactive power theory is greater than before due to the increased interest to use active power filters to compensate the non-sinusoidal currents produced by nonlinear loads. However, this theory can only be applied to three-phase systems of three or four conductors. This paper presents a new definition of instantaneous power that can be applied to all multiphase systems. Thus, the concept of tensor product is introduced to define the instantaneous power tensor and also the current decomposition in active and reactive components regardless of the number of phases of the power system. Finally, an exhaustive analysis of practical cases has been carried out at a simulation level which verified the proposed approach.

Keywords: Tensor analysis, active power filter, instantaneous power, tensor product, multiphase systems.

\section{INTRODUCCIÓN}

Definir apropiadamente las componentes de la potencia tales como potencia activa, potencia reactiva y potencia aparente son, en la actualidad, motivo de discusión y han dado como resultado una gran cantidad de teorías. Las componentes de la potencia están bien definidas bajo condiciones de operación sinusoidal en sistemas monofásicos lineales o en sistemas trifásicos balanceados lineales. Sin embargo, la utilización de dispositivos electrónicos en sectores residenciales, comerciales e industriales ha traído consigo un aumento significativo de las perturbaciones que afectan la calidad de la forma de onda. En este caso, la definición de las componentes de la potencia es controversial admitiendo múltiples

1 Departamento de Ingeniería Eléctrica, Electrónica y Computación. Universidad Nacional de Colombia. Campus La Nubia, A.A. 127. Manizales, Colombia. E-mail: ajustarizf@unal.edu.co; eacanopl@unal.edu.co

2 Departamento de Ingeniería Electrónica. Facultad de Ingeniería. Universidad de Buenos Aires. Paseo Colón 850-1063. Buenos Aires, Argentina. E-mail: htacca@ fi.uba.ar 
enfoques, lo cual ha dado lugar al planteamiento de muchas teorías. No obstante, todas estas teorías dependen del dominio (frecuencia, tiempo o tiempofrecuencia) y del número de fases del sistema eléctrico donde se realiza el análisis.

Como puede observarse en la Tabla 1, desde comienzos del siglo pasado muchas teorías han sido planteadas para resolver el problema [1-11]. Sin embargo, en la actualidad no se ha podido llegar a un consenso sobre una teoría única donde las componentes de potencia en régimen no sinusoidal no sean refutadas.

Tabla 1. Resumen de algunas de las teorías de potencia.

\begin{tabular}{|l|l|l|l|}
\hline \multicolumn{1}{|c|}{ Teoría } & \multicolumn{1}{c|}{ Dominio } & \multicolumn{1}{c|}{ Sistema } & Año \\
\hline I. Budeanu [1] & Frecuencia & Monofásico & 1927 \\
\hline S. Fryze [1] & Tiempo & Monofásico & 1931 \\
\hline M. Depenbrock [2] & Tiempo & Polifásico & 1962 \\
\hline H. Akagi [3] & Tiempo & Trifásico & 1983 \\
\hline L.S. Czarnecki [4] & Frecuencia & Trifásico & 1988 \\
\hline J.L. Willems [5] & Tiempo & Polifásico & 1992 \\
\hline F.Z. Peng [6] & Tiempo & Trifásico & 1996 \\
\hline W. Yoon [7] & $\begin{array}{l}\text { Tiempo- } \\
\text { Frecuencia }\end{array}$ & Monofásico & 2000 \\
\hline E.A.C. Plata [8] & $\begin{array}{l}\text { Tiempo- } \\
\text { Frecuencia }\end{array}$ & Trifásico & 2003 \\
\hline X. Dai [9] & Tiempo & Polifásico & 2004 \\
\hline P. Salmerón [10] & Tiempo & Polifásico & 2009 \\
\hline A.J. Ustariz [11] & Tiempo & Polifásico & 2010 \\
\hline
\end{tabular}

Inicialmente, las teorías se basaban en el concepto del valor medio y eran implementadas principalmente para la medida del contenido de potencia armónica. Pero fue en 1983 cuando Akagi, Kanazawa y Nabae [3] introdujeron la teoría de la potencia reactiva instantánea, basada en el concepto de valor instantáneo, para formas de onda arbitrarias, incluyendo estados transitorios.

La teoría de la potencia reactiva instantánea ha sido el tema de varias interpretaciones y mejoras [5-6], [8], lo que ha permitido destacarla como la más usada en el cálculo de las corrientes de referencia de los llamados filtros activos de potencia (APFs "active power filters"). Sin embargo, esta teoría solamente puede ser aplicada en sistemas trifásicos de tres y cuatro conductores.

Por otra parte, en las teorías relacionadas a la transferencia de la potencia en sistemas polifásicos, en la literatura se propone la descomposición de la corriente en dos componentes ortogonales [2], [5]. La primera componente se obtiene a partir del concepto de potencia activa instantánea, mientras que la segunda componente se calcula como el complemento de la anterior componente para obtener la corriente total. Por tal motivo estas teorías no presentan una definición formal de la potencia reactiva instantánea en sistemas de $\mathrm{n}$-fases.

A pesar de esto, en la última década un importante avance para definir el concepto de potencia reactiva en sistemas polifásicos es propuesto en [9-10]. Este nuevo enfoque define la potencia reactiva instantánea en sistemas polifásicos partiendo de la definición del producto exterior en términos del producto tensorial. Sin embargo, no existe una relación recíproca entre el operador introducido para definir las componentes de la corriente y cada término de la potencia.

En este artículo, una definición integral de la potencia instantánea en sistemas polifásicos es propuesta. La herramienta matemática utilizada también permite definir formalmente la potencia reactiva instantánea sin importar el número de fases del sistema de potencia. Además, se especifican las estrategias de control para la obtención de las corrientes de referencia de los APFs en sistemas polifásicos. Finalmente, se han implementado a nivel de simulación una serie de casos prácticos con los cuales se ha validado el enfoque propuesto.

\section{TEORÍA GENERALIZADA DE LA POTENCIA INSTANTÁNEA}

En la actualidad una moderna notación está siendo utilizada en los sistemas eléctricos polifásicos (ver Figura 1), donde las tensiones instantáneas y las corrientes instantáneas son expresadas como vectores espaciales.

En un sistema de $\mathrm{n}$-fases los vectores espaciales de tensión y corriente están dados por:

$$
\vec{v}=\left[\begin{array}{c}
v_{1} \\
v_{2} \\
\vdots \\
v_{n}
\end{array}\right] ; \quad \vec{i}=\left[\begin{array}{c}
i_{1} \\
i_{2} \\
\vdots \\
i_{n}
\end{array}\right]
$$




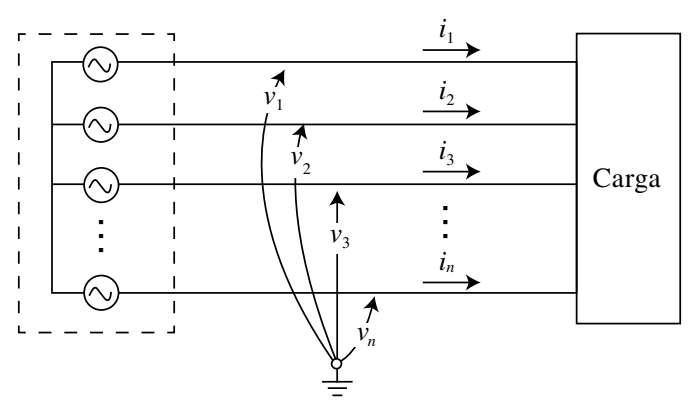

Figura 1. Sistema eléctrico de n-fases.

La formulación propuesta utiliza la representación vectorial de la tensión y la corriente para definir una nueva expresión de la potencia instantánea, denominada "tensor de potencia instantánea" [12]. Esta expresión es obtenida mediante el producto tensorial entre los vectores de tensión y corriente así:

$$
\wp_{i j}=\vec{v} \otimes \vec{i}=\vec{v} \vec{i}^{t}
$$

donde, $\wp_{i j}$ es el tensor instantáneo de potencia y el superíndice $t$ hace referencia al operador de transposición. Por lo tanto, al reemplazar (1) en (2), el tensor instantáneo de potencia es igual a:

$$
\wp_{i j}=\left[\begin{array}{cccc}
v_{1} i_{1} & v_{1} i_{2} & \cdots & v_{1} i_{n} \\
v_{2} i_{1} & v_{2} i_{2} & \cdots & v_{2} i_{n} \\
\vdots & \vdots & \ddots & \vdots \\
v_{n} i_{1} & v_{n} i_{2} & \cdots & v_{n} i_{n}
\end{array}\right]
$$

Por otra parte, al aplicar el operador de transposición en (2) se obtiene el tensor de potencia instantáneo transpuesto, así:

$$
\wp_{i j}^{t}=\left(\vec{v} \vec{i}^{t}\right)^{t}=\vec{i} \vec{v}^{t}=\vec{i} \otimes \vec{v}
$$

Esta expresión implica que el vector de corriente puede ser obtenido desde la definición del tensor instantáneo de potencia así:

$$
\vec{i}=\frac{\wp_{i j}^{t}}{\|\vec{v}\|^{2}} \vec{v}
$$

Aquí $\|\cdot\|$ denota la norma Euclidiana de vectores. Ahora, partiendo de la siguiente igualdad:

$$
\wp_{i j}^{t}=\wp_{i j}+\left(\wp_{i j}^{t}-\wp_{i j}\right)
$$

y reemplazando (6) en (5), el vector de corriente puede expresarse como:

$$
\vec{i}=\frac{\wp_{i j}}{\|\vec{v}\|^{2}} \vec{v}+\frac{\wp_{i j}^{t}-\wp_{i j}}{\|\vec{v}\|^{2}} \vec{v}
$$

El primer término es la corriente activa instantánea:

$$
\begin{aligned}
\vec{i}_{p} & =\frac{\wp_{i j}}{\|\vec{v}\|^{2}} \vec{v} \\
& =\frac{1}{\|\vec{v}\|^{2}}\left[\begin{array}{cccc}
v_{1} i_{1} & v_{1} i_{2} & \cdots & v_{1} i_{n} \\
v_{2} i_{1} & v_{2} i_{2} & \cdots & v_{2} i_{n} \\
\vdots & \vdots & \ddots & \vdots \\
v_{n} i_{1} & v_{n} i_{2} & \cdots & v_{n} i_{n}
\end{array}\right]\left[\begin{array}{c}
v_{1} \\
v_{2} \\
\vdots \\
v_{n}
\end{array}\right] \\
& =\frac{1}{\|\vec{v}\|^{2}}\left[\begin{array}{c}
v_{1} i_{1} v_{1}+v_{1} i_{2} v_{2}+\cdots+v_{1} i_{n} v_{n} \\
v_{2} i_{1} v_{1}+v_{2} i_{2} v_{2}+\cdots+v_{2} i_{n} v_{n} \\
\vdots \\
v_{n} i_{1} v_{1}+v_{n} i_{2} v_{2}+\cdots+v_{n} i_{n} v_{n}
\end{array}\right] \\
& =\frac{\left(v_{1} i_{1}+v_{2} i_{2}+\cdots+v_{n} i_{n}\right)}{\|\vec{v}\|^{2}}\left[\begin{array}{c}
v_{1} \\
v_{2} \\
\vdots \\
v_{n}
\end{array}\right]
\end{aligned}
$$

Este término representa la proyección del vector de corriente sobre el vector de tensión.

Por lo tanto, desde (7) y (8), la corriente reactiva instantánea es expresada como sigue:

$$
\begin{aligned}
\vec{i}_{q} & =\frac{\wp_{i j}^{t}-\wp_{i j}}{\|\vec{v}\|^{2}} \vec{v}=\frac{\wp_{i j}^{t}}{\|\vec{v}\|^{2}} \vec{v}-\frac{\wp_{i j}}{\|\vec{v}\|^{2}} \vec{v} \\
& =\vec{i}-\vec{i}_{p}
\end{aligned}
$$

Este término representa la componente en cuadratura con respecto al vector de tensión.

De forma similar a lo establecido en [5], con estas dos corrientes se pueden definir los conceptos tradicionales de potencia instantánea. Es así como la convencional potencia activa instantánea en sistemas de $n$-fases puede ser definida como el 
producto escalar entre los vectores de tensión y corriente activa instantánea, así:

$$
p=\left\langle\vec{v} \cdot \vec{i}_{p}\right\rangle
$$

El tensor instantáneo de potencia imaginaria definido mediante el producto tensorial entre los vectores de tensión y corriente reactiva instantánea

$$
q_{i j}=\vec{v} \otimes \vec{i}_{q}
$$

permite calcular la norma de la potencia imaginaria instantánea, así:

$$
q=\left\|q_{i j}\right\|=\left\|\vec { v } \left|\left\|\mid \vec{i}_{q}\right\|\right.\right.
$$

y la potencia aparente vectorial instantánea como:

$$
s=\left\|\wp_{i j}\right\|=\sqrt{p^{2}+q^{2}}
$$

\section{CORRIENTE DE REFERENCIA PARA EL CONTROL DE APFS}

\section{Estrategia de compensación instantánea}

Como resultado de la descomposición del vector de corriente dado en (7), es posible plantear una estrategia de compensación desde el punto de vista instantáneo denominada estrategia-ITC, “instantaneous-time compensation”.

El objetivo de esta estrategia es eliminar la componente de corriente que no transmite potencia instantánea, permitiendo minimizar las pérdidas en las líneas sin alterar la transmisión de potencia instantánea.

En este tipo de compensación el vector instantáneo de corrientes de referencia para el control del $A P F$ es obtenido por:

$$
\vec{i}_{-r e f}=\vec{i}_{q}=\frac{\wp_{i j}^{t}-\wp_{i j}}{\|\vec{v}\|^{2}} \vec{v}
$$

y su esquema algorítmico es resumido en la Figura 2, donde se muestran las diferentes operaciones implementadas en Matlab-Simulink (producto tensorial, producto punto, etc.).

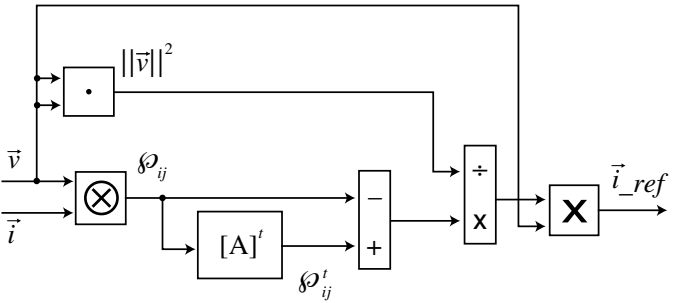

Figura 2. Diagrama de bloques (estrategia-ITC).

\section{Estrategias de compensación promediada}

En régimen permanente, el tensor de potencia instantánea se puede dividir en dos términos, así:

$$
\wp_{i j}=\bar{\wp}_{i j}+\tilde{\wp}_{i j}
$$

donde la barra superior (-) y la tilda superior $(\sim)$ hacen referencia al valor promedio y al término variable del tensor. Tal que,

$$
\bar{\wp}_{i j}=\frac{1}{T} \int_{T} \wp_{i j} d t
$$

Como resultado de la división en régimen permanente del tensor de potencia instantánea, es posible plantear según [13] tres estrategias de compensación vistas desde el lado de la fuente.

La primera estrategia permite obtener potencia activa constante (estrategia-CAP, "constant active power"). La corriente de referencia para el control $\operatorname{del} A P F$ es

$$
\vec{i}_{-r e f}=\vec{i}-\frac{\operatorname{traza}\left(\bar{\wp}_{i j}\right)}{\|\vec{v}\|^{2}} \vec{v}
$$

Con la segunda estrategia se obtiene un factor de potencia igual a la unidad (estrategia-UPF "unity power factor"). En este caso, la corriente de referencia para el control del $A P F$ es

$$
\vec{i}_{-r e f}=\vec{i}-\frac{\operatorname{traza}\left(\bar{\wp}_{i j}\right)}{\frac{1}{T} \int_{T}\|\vec{v}\|^{2} d t} \vec{v}
$$

Finalmente, la tercera estrategia logra una perfecta cancelación de los armónicos de la corriente (estrategia-PHC, "perfect harmonic cancellation"). Ahora, la corriente de referencia para el control $\operatorname{del} A P F$ es 


$$
\vec{i}_{-r e f}=\vec{i}-\frac{\operatorname{traza}\left(\bar{\wp}_{i j}\right)}{\frac{1}{T} \int_{T}\left\|\vec{v}_{f}^{+}\right\|^{2} d t} \vec{v}_{f}^{+}
$$

donde $\vec{v}_{f}^{+}$es el vector de tensiones de secuencia positiva a la frecuencia fundamental.

Los algoritmos para las estrategias de compensación promediada son resumidos en la Figura 3a, donde se muestra el esquema general de las diferentes operaciones implementadas (filtro pasa bajo ( $L P F$, "low pass filter"), traza, etc.). El bloque selector de la estrategia de compensación es presentado en la Figura $3 b$.

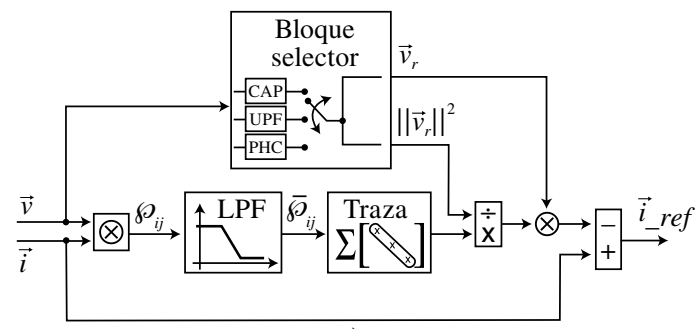

a)

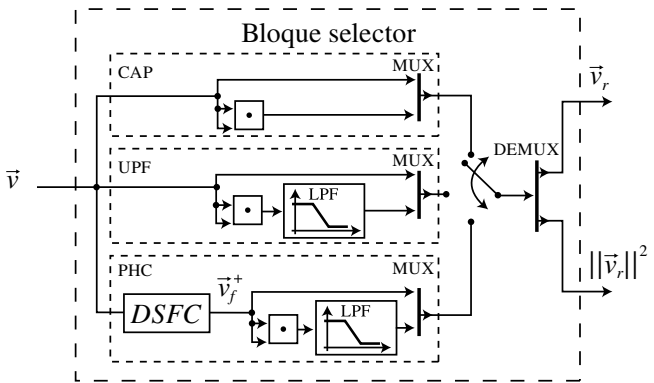

b)

Figura 3. Diagrama de bloques: a) Algoritmo general y b) Bloque selector.

\section{Estrategia de compensación ideal}

En régimen permanente también se puede plantear otra descomposición del tensor instantáneo de potencia, así:

$$
\wp_{i j}={ }^{\text {ideal }} \wp_{i j}+{ }^{\text {no-ideal }} \wp_{i j}
$$

Establecer esta división, del tensor instantáneo de potencia, implica definir un sistema eléctrico ideal como un circuito compuesto de una fuente de tensión sinusoidal y equilibrada (fuente de referencia) alimentando una carga resistiva, balanceada y lineal (carga de referencia). Bajo estas condiciones el tensor de potencia ideal es definido como:

$$
\begin{aligned}
{ }_{\text {ideal }} \wp_{i j} & =\vec{v}_{f}^{+} \otimes \vec{i}_{f}^{+} \\
& =\vec{v}_{f}^{+} \otimes\left(\frac{\operatorname{traza}\left(\bar{\wp}_{i j}\right)}{\left\|\vec{v}_{f}^{+}\right\|^{2}} \vec{v}_{f}^{+}\right)
\end{aligned}
$$

Por otra parte, cualquier situación que produzca una no-conformidad con respecto a esas condiciones ideales se verá reflejada en el término del tensor instantáneo de potencia no-ideal y en consecuencia podrá ser compensada.

Como resultado, es posible plantear en régimen permanente una nueva estrategia de compensación (estrategia-NPC, "nonconformity power cancellation") vista desde el lado de la fuente. En este caso la corriente de referencia es estimada por

$$
\begin{aligned}
\vec{i}_{r e f} & =\frac{{ }^{n o-i d e a l} \wp_{i j}^{t}}{\|\vec{v}\|^{2}} \\
& =\frac{\left(\wp_{i j}-{ }^{i d e a l} \wp_{i j}\right)^{t}}{\|\vec{v}\|^{2}} \vec{v}
\end{aligned}
$$

El esquema algorítmico de la estrategia de compensación -NPC es mostrado en la Figura 4.

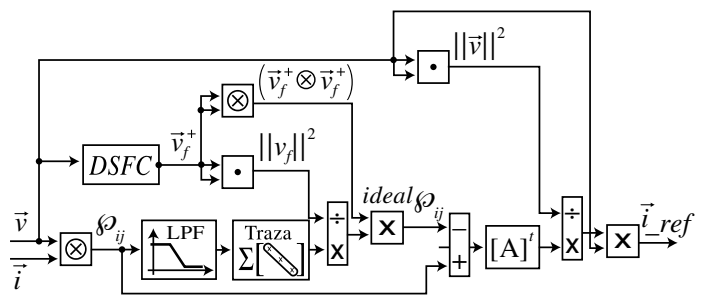

Figura 4. Diagrama de bloques (estrategia-NPC).

\section{COMPONENTE FUNDAMENTAL DE SECUENCIA DIRECTA}

Para calcular la componente instantánea de secuencia directa a frecuencia fundamental se ha implementado el bloque DSFC, "direct sequence fundamental component", el cual admite formas de ondas no sinusoidales, desequilibradas y polifásicas. 
El algoritmo utilizado para implementar el DSFC (ver Figura 5) está basado en el método desarrollado en [14] para estimar la componente fundamental de secuencia directa en sistemas trifásicos.

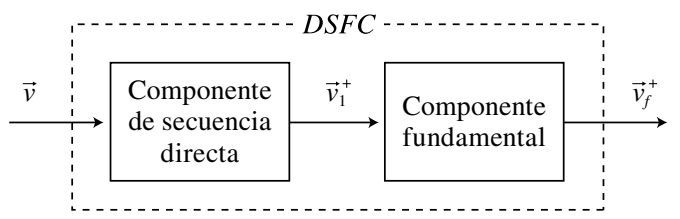

Figura 5. Esquema general del bloque DSFC.

Para determinar la componente de secuencia directa en un sistema de $n$-fases es implementado el bloque componente de secuencia directa, donde la transformación instantánea de Fortescue es aplicada [15]. Esto es,

$$
v_{1}^{+}=\frac{1}{n}\left(v_{1}+a^{-(n-1)} v_{2}+\cdots+a^{-1} v_{n}\right)
$$

Aquí, el operador $a$ es definido como $a=e^{j 2 \pi / n}$, el cual es implementado por un filtro pasa todo ( $A L P F$, "all pass filter"). La función de transferencia del filtro pasa todo es:

$$
F(s)=\frac{s-z}{s+p}
$$

Aquí, se cumple que:

$$
z=p=\sqrt{\frac{\omega^{2}[1-\cos (2 \pi / n)]}{\cos (2 \pi / n)+1}}
$$

donde $\omega$ es la frecuencia fundamental en rad/s. En la Figura 6 se muestra cómo el desfase impuesto por el valor de la potencia negativa del operador $a$ es obtenido por la conexión en cascada de varios $A L P F$.

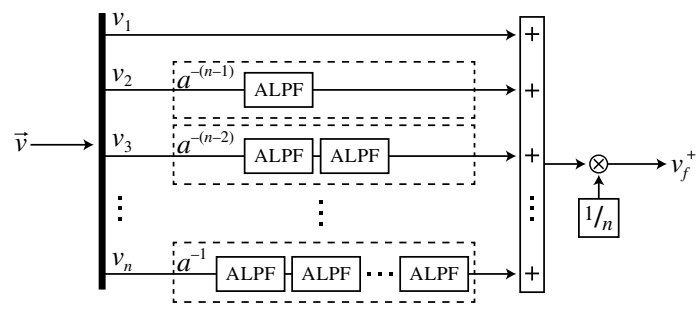

Figura 6. Método para estimar la componente de secuencia directa.

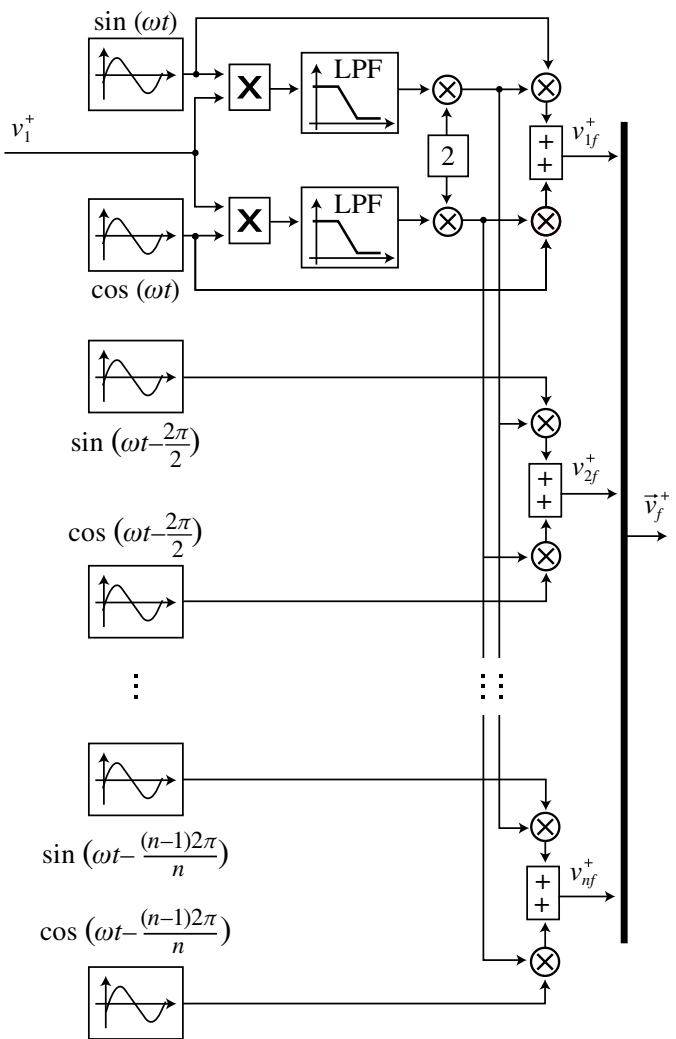

Figura 7. Método para estimar la componente de frecuencia fundamental.

El procedimiento para determinar la componente fundamental se basa en el hecho de que la forma de onda periódica $v_{1}^{+}$(sin componente dc), se expresa en series de Fourier de la siguiente forma:

$$
\begin{aligned}
v_{1}^{+}= & V_{11}^{+} \sin \left(\omega t+\theta_{11}\right) \\
& +V_{12}^{+} \sin \left(2 \omega t+\theta_{12}\right) \\
& \vdots \\
& +V_{1 n}^{+} \sin \left(n \omega t+\theta_{1 n}\right)
\end{aligned}
$$

Al multiplicar la expresión (26) por sin $(\omega t)$ y calcular su valor promedio, se tiene lo siguiente:

$$
\frac{1}{T} \int_{T} v_{1}^{+} \sin (\omega t)=\frac{1}{2} V_{11}^{+} \cos \left(\theta_{11}\right)
$$

Mientras que, si (26) se multiplica por cos $(\omega t)$ y se calcula su valor promedio, el resultado es:

$$
\frac{1}{T} \int_{T} v_{1}^{+} \cos (\omega t)=\frac{1}{2} V_{11}^{+} \sin \left(\theta_{11}\right)
$$


Ahora, si (27) y (28) se multiplican por 2 y cada resultado se multiplica por $\sin (\omega t)$ y $\cos (\omega t)$ y luego se suman, se obtiene el valor fundamental. Esto es,

$$
v_{1 f}^{+}=V_{11}^{+}\left[\cos \left(\theta_{11}\right) \sin (\omega t)+\sin \left(\theta_{11}\right) \cos (\omega t)\right]
$$

Los otros términos $v_{2 f}^{+}, v_{3 f}^{+}, \ldots, v_{n f}^{+}$son calculados de forma similar utilizando funciones sinusoidales desfasadas tal como se muestra en la Figura 7.

Por lo tanto, la componente fundamental de la tensión de secuencia directa se puede estimar instantáneamente utilizando solamente filtros pasa bajo, multiplicadores y sumadores.

\section{COMPENSACIÓN DE CARGAS POLIFÁSICAS: RESULTADOS DE SIMULACIÓN}

Para ilustrar la teoría tensorial propuesta, las cinco estrategias de compensación han sido aplicadas a los siguientes cuatro sistemas eléctricos polifásicos: a) sistema bifásico trifilar, b) sistema trifásico trifilar, c) sistema trifásico tetrafilar y d) sistema hexafásico. En cada sistema eléctrico simulado la misma fuente de tensión trifásica, no-sinusoidal y desequilibrada (ver sus parámetros en la Tabla 2) alimenta un tipo de carga no-lineal y polifásica a través de un transformador ideal "TRF" con una conexión específica.

Tabla 2. Parámetros de la fuente de tensión trifásica.

\begin{tabular}{|c|c|c|c|c|}
\hline \multicolumn{5}{|c|}{ Fuente de tensión distorsionada } \\
\hline Fase & fundamental & $\mathrm{h}=3$ & $\mathrm{~h}=5$ & $\mathrm{~h}=7$ \\
\hline $\mathrm{V}_{\mathrm{a}}$ & $13.212 \angle 0^{\circ}$ & $357 \angle 0^{\circ}$ & $528 \angle 0^{\circ}$ & $634 \angle 0^{\circ}$ \\
\hline $\mathrm{V}_{\mathrm{b}}$ & $12.324 \angle-120^{\circ}$ & $333 \angle 0^{\circ}$ & $493 \angle 120^{\circ}$ & $592 \angle-120^{\circ}$ \\
\hline $\mathrm{V}_{\mathrm{c}}$ & $14.070 \angle 120^{\circ}$ & $380 \angle 0^{\circ}$ & $563 \angle-120^{\circ}$ & $675 \angle 120^{\circ}$ \\
\hline
\end{tabular}

Los circuitos mostrados a continuación han sido implementados en Matlab-Simulink. En todos los casos, el filtro activo de potencia es modelado como una fuente de corriente ideal controlada por la corriente de referencia obtenida con el enfoque propuesto. Además, $t=0,1 \mathrm{~s}$ es el tiempo en el cual se conecta el filtro activo de potencia al sistema. La información mostrada en las siguientes figuras es organizada como sigue: (a) Tensión medida en los terminales de la fuente de alimentación $v_{S}$

(b) Tensión medida en los terminales del devanado secundario del transformador $v_{T}$

(c) corrientes medidas en el lado de la fuente de alimentación $i_{S_{-} I T C}, i_{S_{-} C A P}, i_{S_{-} U P F}, i_{S_{-} P H C}, i_{S_{-} N P C}$.

(d) corrientes medidas en el lado secundario del transformador $i_{T_{-} I T C}, i_{T_{-} C A P}, i_{T_{-} U P F}, i_{T_{-} P H C}$, $i_{T_{-} N P C}$.

Los términos relacionados con los índices tradicionales para medir la calidad de potencia presentados en las Tablas 3, 4, 5 y 6 están basados en factores individuales definidos en [16] para sistemas trifásicos, no-sinusoidales y desbalanceados, así:

Distorsión armónica total de tensión:

$T H D_{V}=\frac{V_{e H}}{V_{e 1}}=\left[\frac{\sum_{h \neq 1}\left[{ }^{+} V_{h}^{2}+{ }^{-} V_{h}^{2}+\frac{1}{2}\left({ }^{0} V_{h}^{2}\right)\right]}{{ }^{+} V_{1}^{2}+{ }^{-} V_{1}^{2}+\frac{1}{2}\left({ }^{0} V_{1}^{2}\right)}\right]^{\frac{1}{2}}$

Distorsión armónica total de corriente:

$T H D_{I}=\frac{I_{e H}}{I_{e 1}}=\left[\frac{\sum_{h \neq 1}\left[{ }^{+} I_{h}^{2}+{ }^{-} I_{h}^{2}+4\left({ }^{0} I_{h}^{2}\right)\right]}{{ }^{+} I_{1}^{2}+{ }^{-} I_{1}^{2}+4\left({ }^{0} I_{1}^{2}\right)}\right]^{\frac{1}{2}}$

Factor de desbalance en la tensión:

$$
U F_{V}=\left[\frac{V_{e 1}^{2}-{ }^{+} V_{1}^{2}}{V_{e 1}^{2}}\right]^{\frac{1}{2}}=\left[\frac{{ }^{-} V_{1}^{2}+\frac{1}{2}\left({ }^{0} V_{1}^{2}\right)}{{ }^{+} V_{1}^{2}+{ }^{-} V_{1}^{2}+\frac{1}{2}\left({ }^{0} V_{1}^{2}\right)}\right]^{\frac{1}{2}}(32)
$$

Factor de desbalance en la corriente:

$$
U F_{I}=\left[\frac{I_{e 1}^{2}-{ }^{+} I_{1}^{2}}{I_{e 1}^{2}}\right]^{\frac{1}{2}}=\left[\frac{{ }^{-} I_{1}^{2}+4\left({ }^{0} I_{1}^{2}\right)}{{ }^{+} I_{1}^{2}+{ }^{-} I_{1}^{2}+4\left({ }^{0} I_{1}^{2}\right)}\right]^{\frac{1}{2}}
$$

Factor de desplazamiento de fase:

$$
O C F=\left|\frac{\sum_{k=1,2,3} I_{k_{-} 1} \sin \left(\varphi_{k_{-} 1}\right)}{\sum_{k=1,2,3} I_{k_{-} 1}}\right|
$$


El término relacionado con el indicador que integralmente evalúa la calidad de potencia está basado en el factor de desviación de calidad de potencia propuesto por los autores en [17], así:

$$
D F_{p q}=\left[\frac{1}{T} \int_{T}\left(\frac{\sum_{i=1}^{n} \sum_{j=1}^{n}\left(\wp_{i j}-{ }^{\text {ideal }} \wp_{i j}\right)^{2}}{\sum_{i=1}^{n} \sum_{j=1}^{n}{ }_{\text {ideal }}^{2} \wp_{i j}^{2}}\right) d t\right]^{\frac{1}{2}}
$$

\section{Caso $\mathrm{N}^{\circ}$ 1: Sistema bifásico trifilar}

En el sistema bifásico trifilar que se muestra en la Figura 8 se han implementado dos rectificadores monofásicos como carga del circuito eléctrico. Esta carga es alimentada desde un transformador ideal en conexión Scott.

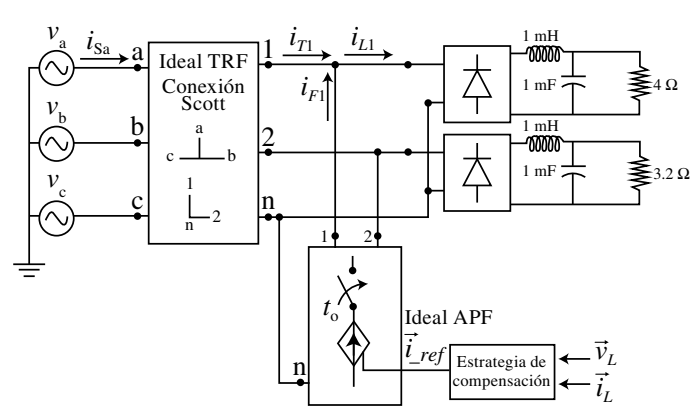

Figura 8. Sistema bifásico trifilar.

Los resultados de la simulación referentes a las formas de ondas de las tensiones y corrientes antes y después de la compensación en el sistema bifásico trifilar, son ilustrados en la Figura 9 y resumidos en la Tabla 3.

Tabla 3. Resumen de los resultados, caso $\mathrm{N}^{\circ} 1$.

\begin{tabular}{|c|c|c|c|c|c|c|}
\hline \multirow{2}{*}{ Factor [\%] } & \multicolumn{7}{|c|}{ Estrategia de compensación } \\
\cline { 2 - 7 } & Antes & $I T C$ & $C A P$ & $U P F$ & $P H C$ & $N P C$ \\
\hline Lado de la fuente de alimentación \\
\hline$T_{H} D \mathrm{~V}$ & 6,53 & 6,53 & 6,53 & 6,53 & 6,53 & 6,53 \\
\hline$T H D_{I}$ & 64,66 & 58,90 & 7,33 & 6,25 & 0,00 & 1,98 \\
\hline$U F_{V}$ & 4,67 & 4,67 & 4,67 & 4,67 & 4,67 & 4,67 \\
\hline$U F_{I}$ & 7,73 & 6,09 & 0,11 & 3,80 & 0,02 & 1,84 \\
\hline$O C F$ & 6,19 & 0,17 & 0,04 & 0,04 & 0,04 & 0,04 \\
\hline$D F_{p q}$ & 66,68 & 57,96 & 10,73 & 11,91 & 8,69 & 8,33 \\
\hline Lado secundario del transformador \\
\hline DF \\
\hline 69
\end{tabular}

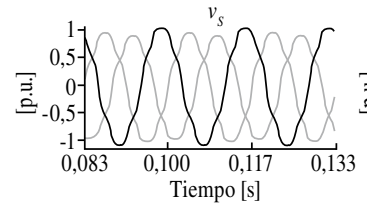

a)
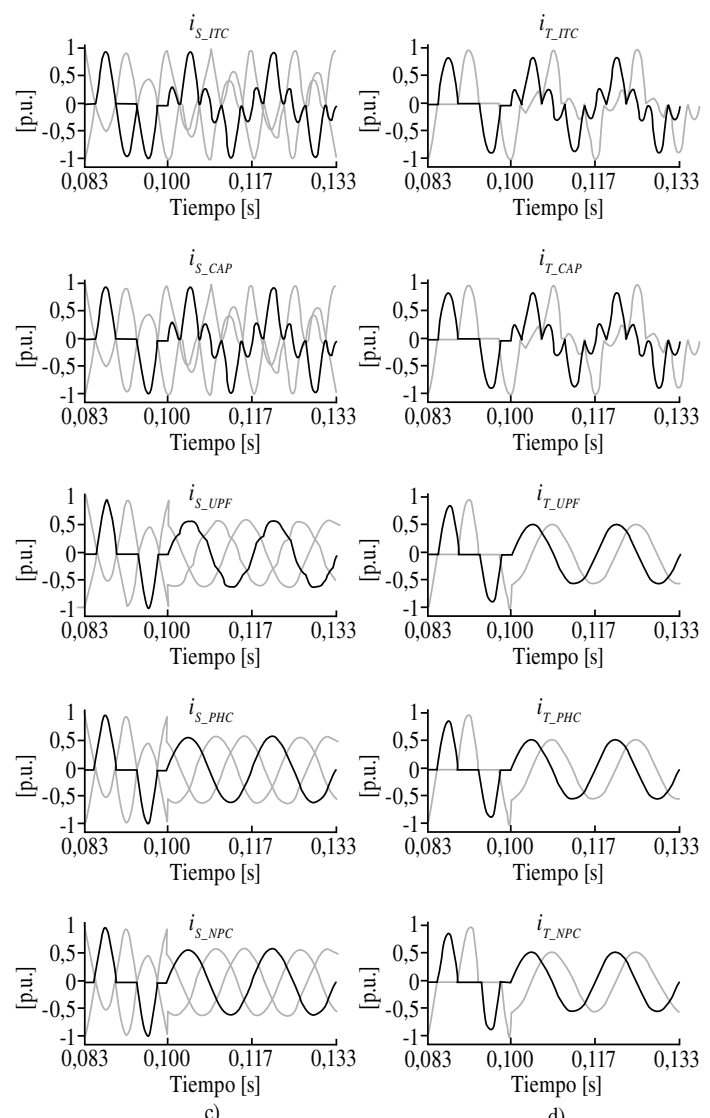

Figura 9. Formas de ondas obtenidas para el caso $\mathrm{N}^{\circ} 1:$ a) $v_{S}$, b) $v_{T}$, c) $i_{S}$, d) $i_{T}$.

En la Tabla 3 se muestran las mediciones de los índices realizadas en la fuente y el secundario del transformador en conexión Scott.

\section{Caso $\mathbf{N}^{\circ}$ 2: Sistema trifásico trifilar}

La carga trifásica mostrada en la Figura 10 es caracterizada por un convencional rectificador trifásico en puente completo con filtro capacitivo en el lado dc. Esta carga es alimentada desde un transformador ideal en conexión $\Delta-Y_{n}$. 


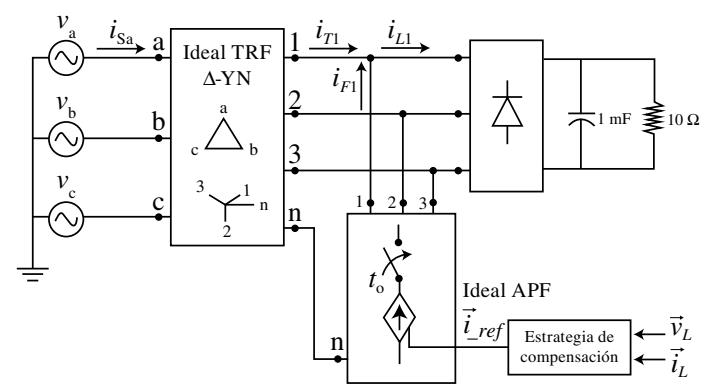

Figura 10. Sistema trifásico trifilar con APF ideal.

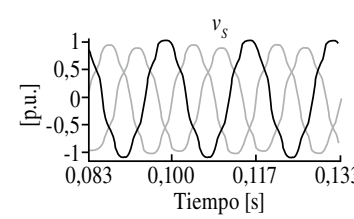

a)
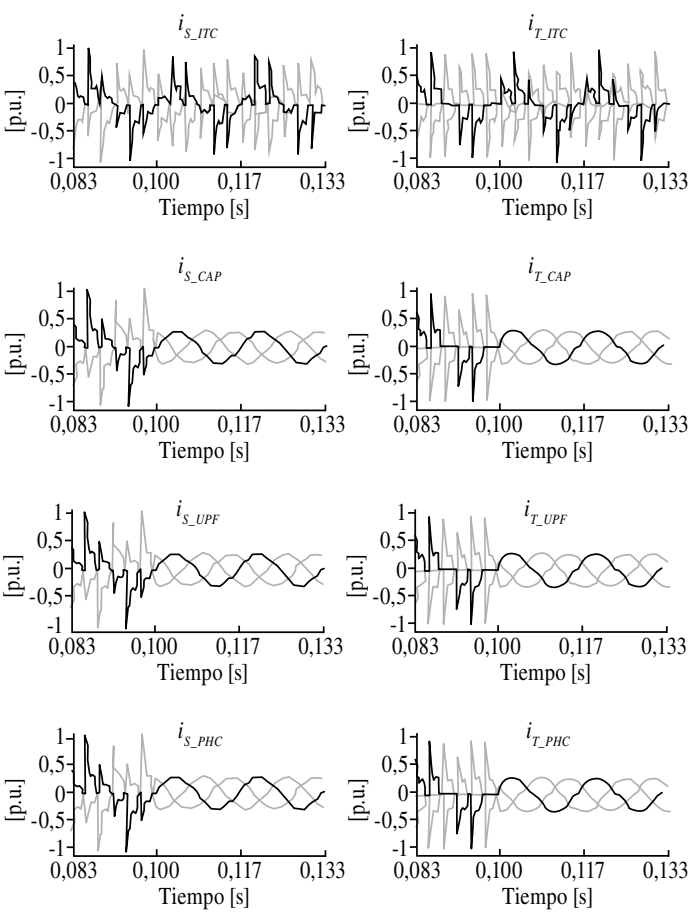

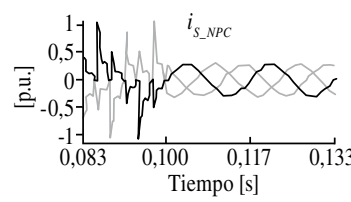

c)
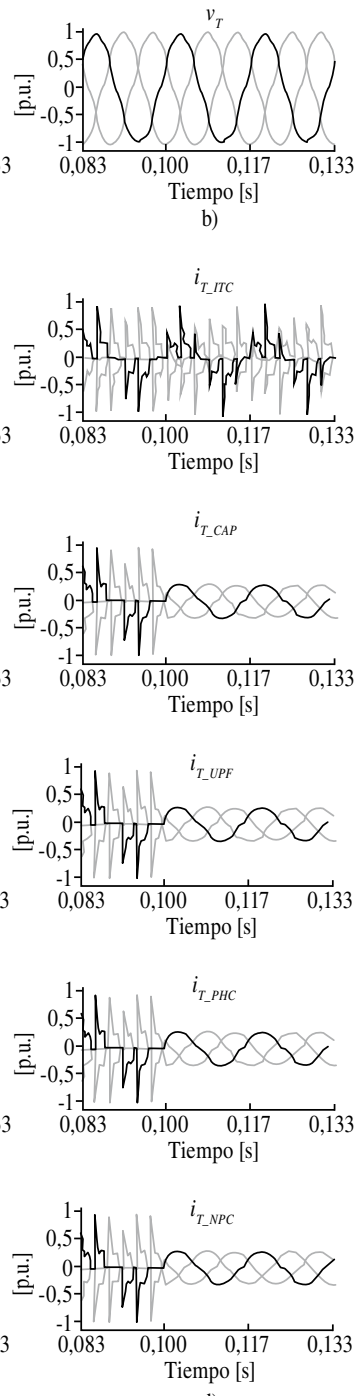

d)

Figura 11. Formas de ondas obtenidas para el caso $\mathrm{N}^{\mathrm{o}} 2$ : a) $v_{S}$, b) $v_{T}$, c) $i_{S}$, d) $i_{T}$.
Los resultados referentes a las formas de ondas de las tensiones y corrientes antes y después de la compensación se muestran en la Figura 11 y se resumen en la Tabla 4. Aquí es posible calcular los indicadores de calidad, tanto en la fuente como en el secundario del transformador.

Tabla 4. Resumen de los resultados, caso $\mathrm{N}^{\circ} 2$.

\begin{tabular}{|c|c|c|c|c|c|c|}
\hline \multirow{2}{*}{ Factor $[\%]$} & \multicolumn{6}{|c|}{ Estrategia de compensación } \\
\hline & Antes & ITC & $C A P$ & $U P F$ & PHC & $N P C$ \\
\hline \multicolumn{7}{|c|}{ Lado de la fuente de alimentación } \\
\hline$T H D_{V}$ & 6,53 & 6,53 & 6,53 & 6,53 & 6,53 & 6,53 \\
\hline$T H D_{I}$ & 91,88 & 84,32 & 7,33 & 6,25 & 0,00 & 1,98 \\
\hline$U F_{V}$ & 4,67 & 4,67 & 4,67 & 4,67 & 4,67 & 4,67 \\
\hline$U F_{I}$ & 12,22 & 12,66 & 0,12 & 3,82 & 0,00 & 1,82 \\
\hline$O C F$ & 18,13 & 3,41 & 0,00 & 0,00 & 0,00 & 0,00 \\
\hline$D F_{p q}$ & 97,27 & 87,43 & 10,73 & 11,92 & 8,70 & 8,33 \\
\hline \multicolumn{7}{|c|}{ Lado secundario del transformador } \\
\hline$T H D_{V}$ & 6,25 & 6,25 & 6,25 & 6,25 & 6,25 & 6,25 \\
\hline$T H D_{I}$ & 91,88 & 84,32 & 7,33 & 6,25 & 0,00 & 1,98 \\
\hline$U F_{V}$ & 3,82 & 3,82 & 3,82 & 3,82 & 3,82 & 3,82 \\
\hline$U F_{I}$ & 12,22 & 12,66 & 0,12 & 3,82 & 0,00 & 1,82 \\
\hline$O C F$ & 18,31 & 3,63 & 0,00 & 0,00 & 0,00 & 0,00 \\
\hline$D F_{p q}$ & 97,13 & 87,29 & 9,61 & 11,01 & 7,33 & 6,81 \\
\hline
\end{tabular}

\section{Caso $\mathbf{N}^{\circ}$ 3: Sistema trifásico tetrafilar}

En la Figura 12 la carga no-lineal es un regulador de corriente-alterna desbalanceado alimentado desde un transformador ideal en conexión $\Delta-Y_{n}$.

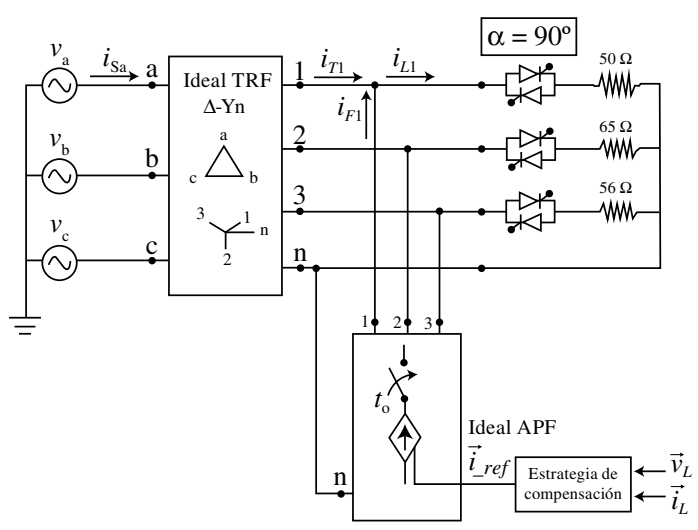

Figura 12. Sistema trifásico tetrafilar con APF ideal.

Los resultados de simulación para las diferentes estrategias de compensación en el sistema trifásico tetrafilar son presentados en la Figura 13. Los resultados de los índices medidos durante la simulación son resumidos en la Tabla 5. 


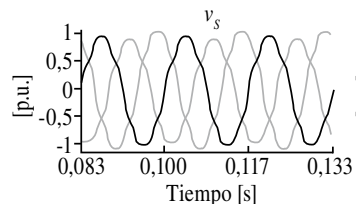

a)
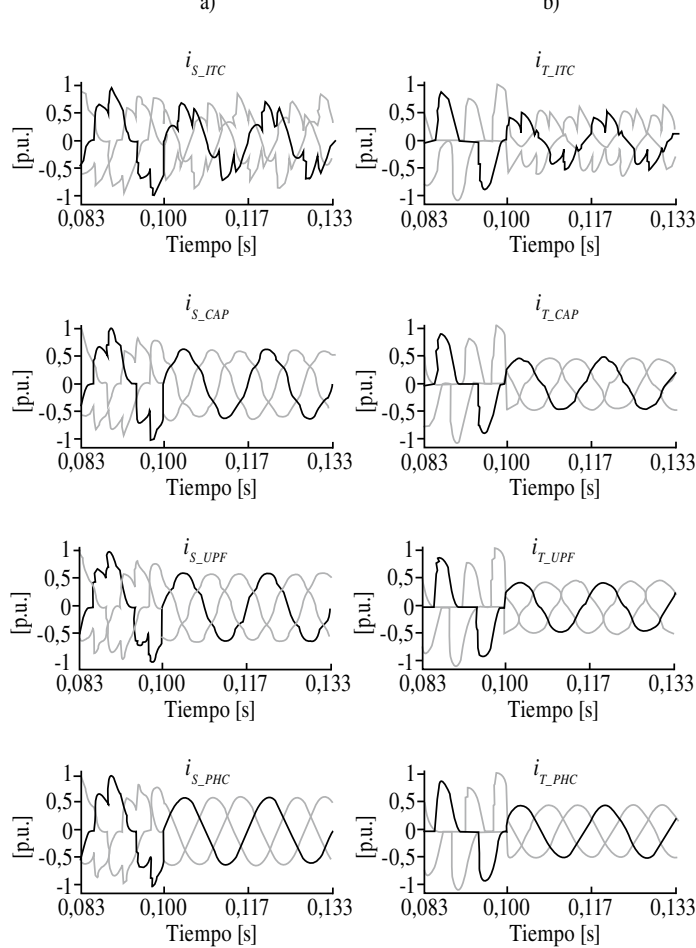

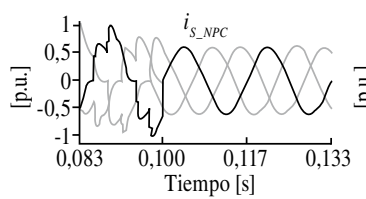

c)

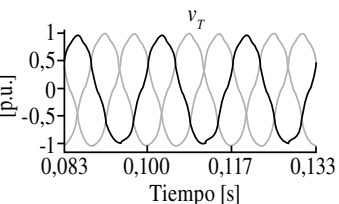

b)

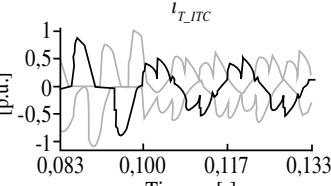

Tiempo $[\mathrm{s}]$

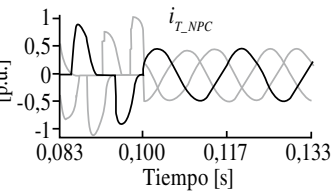

d)
Figura 13. Formas de ondas obtenidas para el caso $\mathrm{N}^{\circ} 3$ : a) $v_{S}$, b) $v_{T}$, c) $i_{S}$, d) $i_{T}$.

Tabla 5. Resumen de los resultados, caso $\mathrm{N}^{\circ} 3$.

\begin{tabular}{|}
\hline \multirow{2}{*}{ Factor [\%] } & \multicolumn{7}{|c|}{ Estrategia de compensación } \\
\cline { 2 - 7 } & Antes & $I T C$ & $C A P$ & $U P F$ & $P H C$ & $N P C$ \\
\hline Lado de la fuente de alimentación \\
\hline$T H D_{V}$ & 6,53 & 6,53 & 6,53 & 6,53 & 6,53 & 6,53 \\
\hline$T H D_{I}$ & 22,17 & 25,40 & 7,33 & 6,25 & 0,00 & 1,98 \\
\hline$U F_{V}$ & 4,67 & 4,67 & 4,67 & 4,67 & 4,67 & 4,67 \\
\hline$U F_{I}$ & 10,36 & 9,99 & 0,12 & 3,82 & 0,00 & 1,82 \\
\hline$O C F$ & 61,86 & 0,72 & 0,00 & 0,00 & 0,00 & 0,00 \\
\hline$D F_{p q}$ & 85,94 & 29,56 & 10,73 & 11,92 & 8,69 & 8,33 \\
\hline
\end{tabular}

\begin{tabular}{|c|c|c|c|c|c|c|c|}
\hline \multirow{2}{*}{ Factor [\%] } & \multicolumn{7}{|c|}{ Estrategia de compensación } \\
\cline { 2 - 7 } & Antes & $I T C$ & $C A P$ & $U P F$ & $P H C$ & NPC \\
\hline Lado secundario del transformador \\
\hline$T H D_{V}$ & 6,25 & 6,25 & 6,25 & 6,25 & 6,25 & 6,25 \\
\hline$T H D_{I}$ & 111,21 & 25,40 & 7,33 & 6,25 & 0,00 & 1,98 \\
\hline$U F_{V}$ & 3,82 & 3,82 & 3,82 & 3,82 & 3,82 & 3,82 \\
\hline$U F_{I}$ & 21,27 & 9,99 & 0,12 & 3,82 & 0,00 & 1,82 \\
\hline$O C F$ & 61,78 & 0,64 & 0,00 & 0,00 & 0,00 & 0,00 \\
\hline$D F_{p q}$ & 112,16 & 29,21 & 9,61 & 11,01 & 7,33 & 6,81 \\
\hline
\end{tabular}

\section{Caso $\mathbf{N}^{\circ}$ 4: Sistema hexafásico}

En el circuito hexafásico de la Figura 14, un rectificador en medio puente ha sido implementado como carga, la cual, es alimentada desde un transformador en conexión $\Delta$-doble Y.

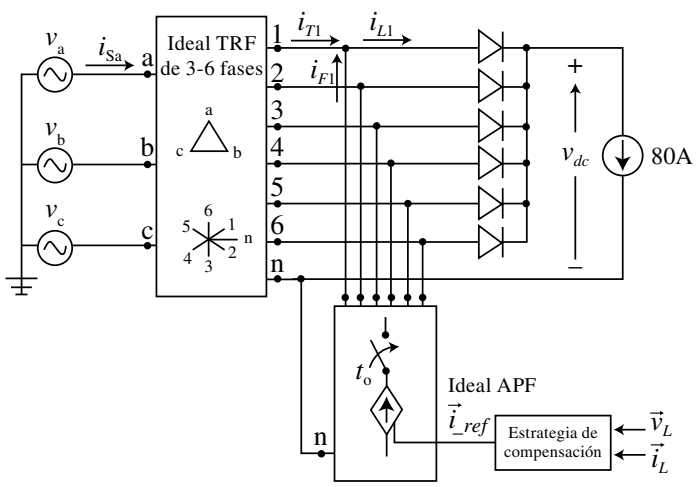

Figura 14. Sistema hexafásico con APF ideal.

La Figura 15 ilustra las formas de ondas, mientras la Tabla 6 lista los resultados de los índices de calidad para el caso $\mathrm{N}^{\circ} 4$.

Tabla 6. Resumen de los resultados, caso $\mathrm{N}^{\circ} 4$.

\begin{tabular}{|c|c|c|c|c|c|c|}
\hline \multirow{2}{*}{ Factor [\%] } & \multicolumn{7}{|c|}{ Estrategia de compensación } \\
\cline { 2 - 7 } & Antes & $I T C$ & $C A P$ & $U P F$ & $P H C$ & $N P C$ \\
\hline Lado de la fuente de alimentación \\
\hline$T H D_{V}$ & 6,53 & 6,53 & 6,53 & 6,53 & 6,53 & 6,53 \\
\hline$T H D_{I}$ & 31,08 & 17,04 & 7,33 & 6,25 & 0,00 & 1,98 \\
\hline$U F_{V}$ & 4,67 & 4,67 & 4,67 & 4,67 & 4,67 & 4,67 \\
\hline$U F_{I}$ & 0,47 & 2,01 & 0,12 & 3,82 & 0,00 & 1,82 \\
\hline$O C F$ & 50,35 & 0,60 & 0,00 & 0,00 & 0,01 & 0,01 \\
\hline$D F_{p q}$ & 67,84 & 19,10 & 10,73 & 11,92 & 8,70 & 8,33 \\
\hline Lado secundario del transformador \\
\hline DF & 182,16 & 18,50 & 9,61 & 11,01 & 7,33 & 6,81 \\
\hline
\end{tabular}

Para los casos analizados, después de conectado el APF, los objetivos de compensación se cumplen según lo estipulado. 


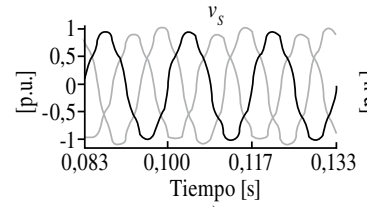

a)
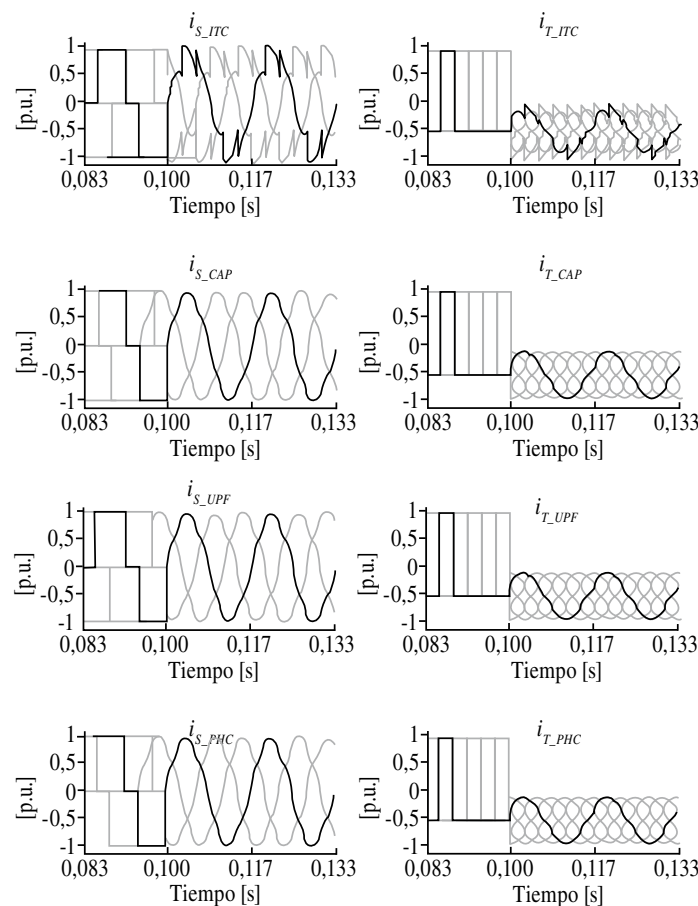

$i_{S N P C}$
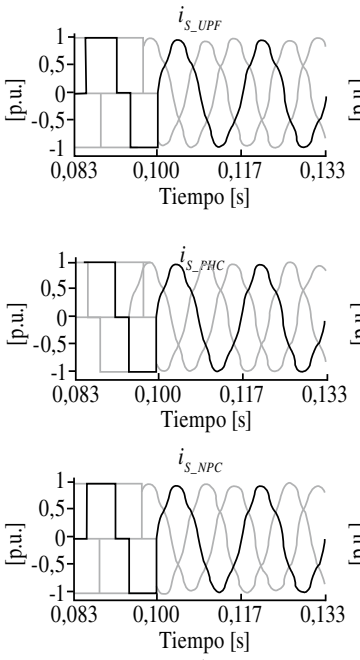
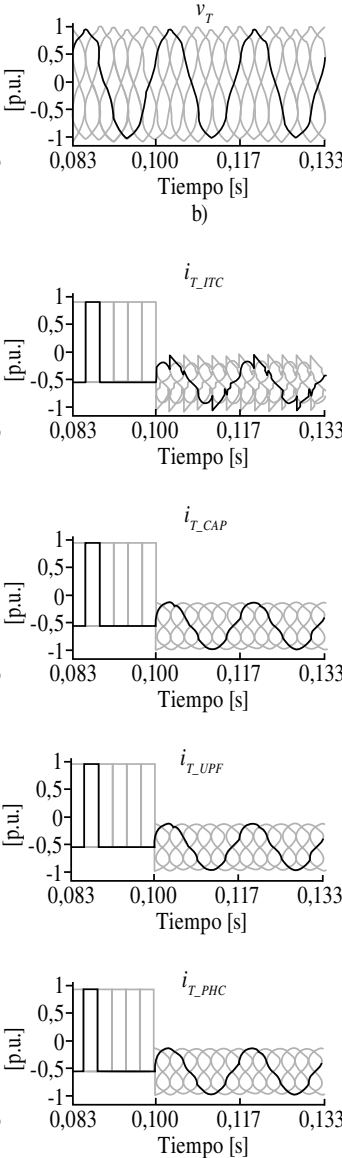

b)

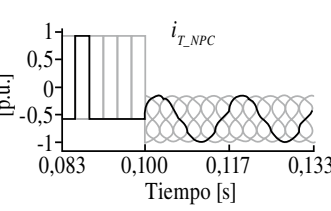

Figura 15. Formas de ondas obtenidas para el caso $\mathrm{N}^{\circ} 4$ : a) $v_{S}$, b) $v_{T}$, c) $i_{S}$, d) $i_{T}$.

Es así como, en la estrategia-ITC el índice individual de calidad que más se minimiza es el OCF. Esta disminución se ve reflejada en los cambios mayores o menores del $D F_{p q}$. Por ejemplo, si el valor del OCF antes de compensar la carga es pequeño, solo se observa un mínimo cambio del $D F_{p q}$ (ver Tablas 3 y 4). Por lo contrario, si el valor del OCF antes de compensar la carga es grande, se observa un gran cambio del $D F_{p q}$ (ver Tablas 5 y 6 ).

Con las estrategias (-CAP, $-U P F,-P H C$ y $-P H C$ ) se logran minimizar todos los índices individuales de calidad y por ende la minimización en mayor proporción del $D F_{p q}$. De todas estas, la estrategia $-P H C$ es la que mejor desempeño presenta en cuanto a la minimización de disturbios individuales. Sin embargo, con la estrategia $-N P C$ es posible obtener el mínimo valor del $D F_{p q}$ (ver Tablas 3, 4, 5 y 6) cuando el sistema eléctrico es afectado por varios disturbios simultáneamente.

\section{CONCLUSIONES}

Una herramienta matemática es introducida para definir de manera integral el concepto de potencia instantánea, partiendo de un producto tensorial entre los vectores de tensión y corriente, independiente del número de fases del sistema eléctrico en estudio. Esta nueva formulación tensorial permite que objetivos de compensación instantáneos, promediados e ideales sean definidos en los sistemas de n-fase. Es así como una novedosa estrategia de control (estrategia-NPC) basada en la eliminación de no-conformidades con respecto a un sistema eléctrico ideal es propuesta. Con esta estrategia es posible obtener el mínimo valor de desviación de la calidad de la potencia debido a disturbios de estado estable. Para lograr este valor mínimo la estrategia $-N P C$ sacrifica la reducción máxima posible en los indicadores individuales de calidad de la energía.

\section{AGRADECIMIENTOS}

Este trabajo fue apoyado en parte por la Universidad Nacional de Colombia, Sede Manizales.

\section{REFERENCIAS}

[1] V.G. Smith. "Reactive and Fictitious Power". Transactions of the American Institute of Electrical Engineers. Vol. 52, Issue 3, pp. 748751. September, 1933. ISSN: 0096-3860. DOI: 10.1109/T-AIEE.1933.5056390.

[2] M. Depenbrock. "Untersuchungen über die Spannungs- und Leis- tungsverhältnisse bei Umrichtern ohne Energiespeicher". Ph.D. thesis. Tech. Univ. Hannover. Hannover, Germany. 1962.

[3] H. Akagi, Y. Kanazawa and A. Nabae. "Instantaneous Reactive Power Compensators Comprising Switching Devices without Energy Storage Components". IEEE Transactions on Industry Applications. Vol. IA-20, Issue 3, pp. 625-630. May, 
1984. ISSN: 0093-9994. DOI: 10.1109/ TIA.1984.4504460.

[4] L.S. Czarnecki. "Orthogonal Decomposition of the Currents in a 3-Phase Nonlinear Asymmetrical Circuits with a Nonsinusoidal Voltage Source". IEEE Transactions on Instrumentation and Measurement. Vol. 37, Issue 1, pp. 30-34. March, 1988. ISSN: 00189456. DOI: 10.1109/19.2658.

[5] J.L. Willems. "A New Interpretation of the Akagi-Nabae Power Components for Nonsinusoidal Three-phase Situations". IEEE Transactions on Instrumentation and Measurement. Vol. 41, Issue 4, pp. 523527. August, 1992. ISSN: 0018-9456. DOI: 10.1109/19.155919.

[6] F.Z. Peng and J.S. Lai. "Generalized instantaneous reactive power theory for threephase power systems". IEEE Transactions on Instrumentation and Measurement. Vol. 45, Issue 1, pp. 293-297. February, 1996. ISSN: 0018-9456. DOI: 10.1109/19.481350.

[7] W.K. Yoon and M.J. Devaney. "Reactive Power measurement using the wavelet transform". IEEE Transactions on Instrumentation and Measurement. Vol. 49, Issue 2, pp. 246252. April, 2000. ISSN: 0018-9456. DOI: 10.1109/19.843057.

[8] E.A.C. Plata and H.E. Tacca. "Three Phase Power Using Wavelet Multiresolution Analysis. Part I: Mathematical Background". IEEE Latin America Transactions. Vol. 1, Issue 1, pp. 15-20. October, 2003. ISSN: 15480992. DOI: 10.1109/TLA.2003.1468614.

[9] X. Dai, G. Liu and R. Gretsch. "Generalized Theory of Instantaneous Reactive Quantity for Multiphase Power System". IEEE Transactions on Power Delivery. Vol. 19, Issue 3, pp. 965-972. July, 2004. ISSN: 08858977. DOI: 10.1109/TPWRD.2004.829914.

[10] P. Salmerón and R.S. Herrera. "Instantaneous Reactive Power Theory-A General Approach to Poly-Phase Systems". Electric Power Systems Research. Vol. 79, Issue 9, pp. 12631270. September, 2009. ISSN: 0378-7796. DOI: 10.1016/j.epsr.2009.03.007
[11] A.J. Ustariz, E.A. Cano and H.E. Tacca. "Tensor Analysis of the Instantaneous Power in Electrical Networks". Electric Power Systems Research. Vol. 80, Issue 7, pp. 788-798. July, 2010. ISSN: 0378-7796. DOI: 10.1016/j.epsr.2009.12.004.

[12] A.J. Ustariz. "Formulación de una Teoría Tensorial de la Potencia Eléctrica: Aplicaciones al Estudio de la Calidad de la Energía". Tesis para optar al grado de doctor. Universidad Nacional de Colombia. Manizales, Colombia. 2011. URL: http://www.bdigital.unal.edu.co/357 8/1/armandojaimeustarizfarfan.2011.pdf

[13] M.I.M. Montero, E.R. Cadaval and F.B. González. "Comparison of Control Strategies for Shunt Active Power Filters in Three-Phase Four-Wire Systems". IEEE Transactions on Power Electronics. Vol. 22, Issue 1, pp. 229236. January, 2007. ISSN: 0885-8993. DOI: 10.1109/TPEL.2006.886616

[14] P. Salmerón and S.P. Litran. "Improvement of the Electric Power Quality Using Series Active and Shunt Passive Filters". IEEE Transactions on Power Delivery. Vol. 25, Issue 2, pp. 10581067. April, 2010. ISSN: 0885-8977. DOI: 10.1109/TPWRD.2009.2034902.

[15] C.L. Fortescue. "Method of Symmetrical Co-Ordinates Applied to the Solution of Polyphase Networks". Transactions of the American Institute of Electrical Engineers. Vol. 37, Issue 2, pp. 1027-1140. July, 1918. ISSN: 0096-3860. DOI: 10.1109/T-AIEE. 1918.4765570.

[16] A.E. Emanuel. "Summary of IEEE Standard 1459: Definitions for the Measurement of Electric Power Quantities under Sinusoidal, Nonsinusoidal, Balanced, or Unbalanced Conditions". IEEE Transactions on Industry Applications. Vol. 40, Issue 3, pp. 869-876. May/June, 2004. ISSN: 0093-9994. DOI: 10.1109/TIA.2004.827452.

[17] A.J. Ustariz, E.A.C. Plata and H.E. Tacca. "New Deviation Factor of Power Quality using Tensor Analysis and Wavelet Packet Transform". International Conference on Power Systems Transients (IPST2011). Delft, Netherlands. June 14-17, 2011. 